\title{
Phase locking and flux-flow resonances in Josephson oscillators driven by homogeneous microwave fields
}

\section{Salerno, Mario; Samuelsen, Mogens Rugholm}

\section{Published in:}

Physical Review B

Link to article, DOI:

10.1103/PhysRevB.59.14653

Publication date:

1999

Document Version

Publisher's PDF, also known as Version of record

Link back to DTU Orbit

Citation (APA):

Salerno, M., \& Samuelsen, M. R. (1999). Phase locking and flux-flow resonances in Josephson oscillators driven by homogeneous microwave fields. Physical Review B, 59(22), 14653-14658.

https://doi.org/10.1103/PhysRevB.59.14653

\section{General rights}

Copyright and moral rights for the publications made accessible in the public portal are retained by the authors and/or other copyright owners and it is a condition of accessing publications that users recognise and abide by the legal requirements associated with these rights.

- Users may download and print one copy of any publication from the public portal for the purpose of private study or research.

- You may not further distribute the material or use it for any profit-making activity or commercial gain

- You may freely distribute the URL identifying the publication in the public portal 


\title{
Phase locking and flux-flow resonances in Josephson oscillators driven by homogeneous microwave fields
}

\author{
M. Salerno* \\ Dipartimento di Scienze Fisiche “E.R.Caianiello," Universita' di Salerno, I-84100 Salerno, Italy \\ M. R. Samuelsen \\ Department of Physics, The Technical University of Denmark, DK-2800 Lyngby, Denmark
}

(Received 21 October 1998)

\begin{abstract}
We investigate both analytically and numerically phase locking and flux-flow resonances of long Josephson junctions in the presence of homogeneous microwave fields. We use a power balance analysis and a perturbation expansion around the uniform rotating solution to derive analytical expressions for $I V$ curves. The dependence of the flux-flow step on the amplitude of the rf field and the appearance of satellite steps are explained. As a result we show that satellite steps around the main flux-flow resonance are spaced by both odd and even harmonics of the rf frequency. An analytical expression for the locking range in current of the phase-lock steps is also derived. These results are found to be in good agreement with numerical results. [S0163-1829(99)14221-8]
\end{abstract}

\section{INTRODUCTION}

Long Josephson oscillators operating in the flux-flow regime have been studied both numerically and experimentally by several groups. ${ }^{1-7}$ The interest in such systems arises mainly from their high output power, wide bandwidth, and tunability features, these being interesting properties for applications in superconducting millimeter-wave electronics. ${ }^{8}$ The dynamical states characterizing flux-flow oscillators manifest themselves in current-voltage (IV) characteristic as high-voltage steps split into a series of equally spaced Fiske substeps. Recently, an analytical description of these singularities in the absence of external microwave fields was provided. ${ }^{9}$ On the other hand, it is known, both from real experiments and numerical simulations, ${ }^{3,4}$ that the application of an external rf field gives rise to "satellite" steps around the main flux-flow resonance which are spaced by harmonics of the applied rf frequency. An analytical explanation of this phenomenon is presently lacking.

The aim of the present paper is to provide a theory for the appearance of satellite steps and phase-locking resonances in the $I V$ characteristic of a Josephson flux-flow oscillator in the case of spatially homogeneous microwave fields. To this end we use both an energy power balance analysis and a perturbative expansion around the uniform rotating background to derive analytical expressions for the $I V$ curve, both in the absence and presence of homogeneous microwave fields. As a result we show that satellite steps appear at positions shifted from the main resonance by both even and odd harmonics of the applied frequency. The heights of these resonances are modulated by the rf field, and can be changed by increasing the amplitude of the microwave field. Moreover, we derive an analytical expression for the locking range in current which extends the one derived in Ref. 10 to arbitrary phase-locking steps. It is worth remarking that the case of the homogeneous rf field investigated here is quite different from the case of the inhomogeneous rf field investigated in Refs. 3 and 4. In this last case, indeed, numerical and experimental results show that only even satellite steps appear in the $I V$ characteristic. This difference suggests a practical way to distinguish the type of coupling (uniform vs boundary) realized in a real experiments.

The paper is organized as follows. In Sec. II we review the model for the flux-flow oscillator, and derive the $I V$ characteristic in the absence of microwave fields by using a power balance analysis (this provides an alternate derivation of the results in Ref. 9). In Sec. III we apply homogeneous microwave fields to the junction, and use a perturbative expansion around the uniform rotating background solution to derive analytical expressions for the $I V$ curve (flux-flow resonances) as well as for the locking ranges of the phaselocked steps. In Sec. IV we compare our analytical expression for the $I V$ characteristic with the results of numerical integrations of the system, obtaining an excellent agreement between theory and numerical experiment. Finally, in Sec. $\mathrm{V}$, we summarize the main results of the paper.

\section{FLUX-FLOW OSCILLATOR}

The electrodynamics of a Josephson junction in the presence of microwaves and magnetic field is described by the perturbed sine Gordon equation

$$
\Phi_{x x}-\Phi_{t t}=\sin (\Phi)+\alpha \Phi_{t}-\eta+\eta_{r f} \sin \left(\Omega t-\theta_{0}\right)
$$

subject to the boundary conditions

$$
\Phi_{x}(0, t)=\Phi_{x}(L, t)=\Gamma
$$

In this equation space and time have been normalized to the Josephson penetration length $\lambda_{J}$ and to the inverse plasma frequency $\omega_{0}^{-1}$, respectively. $\alpha$ is the loss parameter associated to the quasiparticle tunneling, $\eta$ is the normalized $\mathrm{dc}$ bias current, and $\Gamma$ is the normalized magnetic field. A rf field of amplitude $\eta_{r f}$ and frequency $\Omega$ is uniformly applied along the junction, and this may be considered a realistic assumption for overlap and annular geometry. ${ }^{11}$

In this section we concentrate on the main flux-flow resonance, i.e., on the step which arises in absence of micro- 
waves due to the applied magnetic field. The flux-flow regime is characterized by excitations which travel on top of a fast rotating background, so that the effective nonlinearity in the system is drastically reduced. It is then natural to assume a solution of Eq. (1) (with $\eta_{r f}=0$ ) of the form

$$
\Phi(x, t)=\omega t+\Gamma x+\Psi(x, t)+\theta_{1} .
$$

Here $\Psi(x, t)$ denotes a small modulation $(\Psi \ll 1)$ around the rotating background, and $\theta_{1}$ is an arbitrary phase. Inserting Eq. (3) into Eq. (1) and linearizing around $\Phi_{0}=\omega t+\Gamma x$ $+\theta_{1}$, one obtains

$$
\begin{aligned}
\Psi_{x x}-\Psi_{t t}-\alpha \Psi_{t}= & \alpha \omega-\eta+\sin \left(\omega t+\Gamma x+\theta_{1}\right) \\
& +\cos \left(\omega t+\Gamma x+\theta_{1}\right) \Psi,
\end{aligned}
$$

whose solution can be expressed as a Fourier series in space,

$$
\Psi(x, t)=\sum_{n=0}^{\infty}\left[A_{n} \cos (\omega t)+B_{n} \sin (\omega t)\right] \cos \left(k_{n} x\right)
$$

with wave number $k_{n}=(\pi / L) n$. Note that the function $\Psi$ satisfies the boundary condition $\Psi_{x}(0, t)=\Psi_{x}(L, t)=0$, so that Eq. (2) is automatically satisfied. By substituting Eq. (5) into Eq. (4), we obtain

$$
\begin{aligned}
& A_{n}=\frac{2}{1+\delta_{n, 0}} \frac{\left(\omega^{2}-k_{n}^{2}\right) I_{s}(n)+\alpha \omega I_{c}(n)}{\left(\omega^{2}-k_{n}^{2}\right)^{2}+\alpha^{2} \omega^{2}}, \\
& B_{n}=\frac{2}{1+\delta_{n, 0}} \frac{\left(\omega^{2}-k_{n}^{2}\right) I_{c}(n)-\alpha \omega I_{s}(n)}{\left(\omega^{2}-k_{n}^{2}\right)^{2}+\alpha^{2} \omega^{2}},
\end{aligned}
$$

where

$$
\begin{aligned}
& I_{s}(n)=\Gamma L \frac{\left[\cos \left(\theta_{1}\right)-\cos \left(k_{n} L\right) \cos \left(\Gamma L+\theta_{1}\right)\right]}{\left(\Gamma^{2} L^{2}-k_{n}^{2} L^{2}\right)}, \\
& I_{c}(n)=\Gamma L \frac{\left[\sin \left(\Gamma L+\theta_{1}\right) \cos \left(k_{n} L\right)-\sin \left(\theta_{1}\right)\right]}{\left(\Gamma^{2} L^{2}-k_{n}^{2} L^{2}\right)} .
\end{aligned}
$$

In the absence of microwaves ( $\left.\eta_{r f}=0\right)$, the $I V$ characteristic can be computed by introducing the energy of the system,

$$
H=\int_{0}^{L}\left\{\frac{1}{2}\left(\Phi_{t}^{2}+\Phi_{x}^{2}\right)+[1-\cos (\Phi)]\right\} d x,
$$

and performing a power balance analysis around the considered solution. ${ }^{12}$ More precisely, differentiating $H$ with respect to time and using Eq. (1) (with $\eta_{r f}=0$ ), we obtain

$$
\frac{d H}{d t}=\int_{0}^{L}\left[\left(-\alpha \Phi_{t}+\eta\right) \Phi_{t}\right] d x+\left.\Gamma \Phi_{t}\right|_{0} ^{L} .
$$

Power balance is achieved by averaging in time over one period of the rf field and in space over the length of the junction and by imposing the condition $\langle\overline{d H /} d t\rangle=0$ (here and in the following \langle\rangle and the overline denote time and space averages, respectively). We have

$$
-\omega \eta+\alpha \omega^{2}+\alpha\left\langle\overline{\Psi_{t}^{2}}\right\rangle=0,
$$

from which the current voltage characteristic is obtained as

$$
\eta=\alpha \omega\left(1+\frac{1}{2} \sum_{n=0}^{\infty} \frac{A_{n}^{2}+B_{n}^{2}}{1+\delta_{n, 0}}\right) .
$$

In explicit form, using the above expressions for $A_{n}$, and $B_{n}$, we have

$$
\begin{aligned}
\eta= & \alpha \omega+\frac{1}{2} \sum_{n=-\infty}^{\infty}\left(\frac{\Gamma L}{\Gamma L+k_{n} L}\right)^{2} \\
& \times \frac{\sin ^{2}\left(\frac{\Gamma L-k_{n} L}{2}\right)}{\left(\frac{\Gamma L-k_{n} L}{2}\right)^{2}} \frac{\alpha \omega}{\left[\left(\omega^{2}-k_{n}^{2}\right)^{2}+\alpha^{2} \omega^{2}\right]} .
\end{aligned}
$$

Note that here the sum has been extended from $-\infty$ to $\infty$. The first term in Eq. (13) represents the Ohmic part of the $I V$ curve, while the second term is associated with flux-flow. We see that the flux-flow step consists of an infinite series of resonances (Fiske modes) spaced by $\pi / L$ and modulated by a fast Fraunhofer factor which enhances only a few resonances close to $\omega=\Gamma$. Throughout this paper $\alpha L<\pi$, corresponding to low damping. In the limit of infinite length these resonances overlap around $\omega=\Gamma$, and Eq. (13) reduces to the Eck-Scalapino-Taylor result in Ref. 13. It is worth to remark that in Eq. (13) there is no dependence on the phase angle $\theta_{1}$ and that the present derivation based on the power balance analysis is completely equivalent to the one given in Ref. 9 due to the identity $\alpha / \omega\left\langle\overline{\Psi_{t}^{2}}\right\rangle=\langle\overline{\Psi \cos (\omega t+\Gamma x)}\rangle$.

\section{FLUX-FLOW OSCILLATORS IN HOMOGENEOUS MICROWAVE FIELDS}

In this section we consider the case of a nonzero homogeneous rf field applied to the junction. To provide a perturbative expansion for Eq. (1) with $\eta_{r f} \neq 0$, on the background rotation we superimpose a uniform oscillation with the same frequency as the rf field, and consider solutions of the form

$$
\Phi=\Phi_{0}+\Psi(x, t),
$$

with

$$
\Phi_{0}=\omega t+\Gamma x+\beta \sin (\Omega t)+\theta_{1} .
$$

Here $\Psi$ is a small modulation $[\Psi(x, t) \ll 1]$ having both space and time averages equal to zero. We see that the boundary conditions in Eq. (2) are fulfilled if $\Psi(x, t)$ satisfy

$$
\Psi_{x}(0, t)=\Psi_{x}(L, t)=0
$$

Inserting Eq. (14) into Eq. (1), and using the smallness of $\Psi$, we obtain the linearized equation 


$$
\Psi_{x x}-\Psi_{t t}-\alpha \Psi_{t}=\alpha \omega-\eta+\left[\eta_{r f} \cos \left(\theta_{0}\right)-\beta \Omega^{2}\right] \sin (\Omega t)-\left[\eta_{r f} \sin \left(\theta_{0}\right)-\alpha \beta \Omega\right] \cos (\Omega t)+\sin \left(\Phi_{0}\right)+\cos \left(\Phi_{0}\right) \Psi .
$$

To eliminate the explicit dependence on the frequency $\Omega$ from Eq. (17), we choose

$$
\beta=\frac{\eta_{r f}}{\Omega \sqrt{\Omega^{2}+\alpha^{2}}}, \quad \tan \left(\theta_{0}\right)=\frac{\alpha}{\Omega},
$$

so that

$$
\eta_{r f} \cos \left(\theta_{0}\right)-\beta \Omega^{2}=\eta_{r f} \sin \left(\theta_{0}\right)-\alpha \beta \Omega=0
$$

Note that this puts no restriction on the size of $\eta_{r f}$. By expanding the last two terms in Eq. (17), using the Bessel relation

$$
e^{i \beta \sin (\Omega t)}=\sum_{m=-\infty}^{\infty} J_{m}(\beta) e^{i m \Omega t},
$$

and using Eqs. (15)-(18), we can rewrite Eq. (17) in the form

$$
\Psi_{x x}-\Psi_{t t}-\alpha \Psi_{t}=\alpha \omega-\eta+\sum_{m=-\infty}^{\infty} J_{m}(\beta)\left[\sin \left(\Gamma x+\tilde{\omega}_{m} t+\theta_{1}\right)\right]+\sum_{m=-\infty}^{\infty} J_{m}(\beta)\left[\cos \left(\Gamma x+\tilde{\omega}_{m} t+\theta_{1}\right)\right] \Psi
$$

where

$$
\tilde{\omega}_{m}=\omega+m \Omega
$$

To solve Eq. (21) we expand the function $\Psi$ as a double Fourier series of the form

$$
\Psi(x, t)=\sum_{n=0, m=-\infty}^{\infty}\left[A_{n, m} \cos \left(\tilde{\omega}_{m} t\right)+B_{n, m} \sin \left(\tilde{\omega}_{m} t\right)\right] \cos \left(k_{n} x\right),
$$

with $k_{n}=\pi / L n$, so that Eq. (16) is automatically satisfied. By substituting Eq. (23) into Eq. (21) and projecting along the $k_{n}$ and $\tilde{\omega}_{m}$, modes, we obtain

$$
\begin{aligned}
& A_{n, m}=\frac{2}{1+\delta_{n, 0}} \frac{\left(\tilde{\omega}_{m}^{2}-k_{n}^{2}\right) \widetilde{I}_{c}(n, m)+\alpha \tilde{\omega}_{m} \widetilde{I}_{s}(n, m)}{\left(\tilde{\omega}_{m}^{2}-k_{n}^{2}\right)^{2}+\alpha^{2} \tilde{\omega}_{m}^{2}}, \\
& B_{n, m}=\frac{2}{1+\delta_{n, 0}} \frac{\left(\tilde{\omega}_{m}^{2}-k_{n}^{2}\right) \widetilde{I}_{c}(n, m)-\alpha \tilde{\omega}_{m} \widetilde{I}_{s}(n, m)}{\left(\tilde{\omega}_{m}^{2}-k_{n}^{2}\right)^{2}+\alpha^{2} \tilde{\omega}^{2}},
\end{aligned}
$$

with

$$
\begin{aligned}
& \tilde{I}_{s}(n, m)=J_{m}(\beta) I_{s}(n), \\
& \widetilde{I}_{c}(n, m)=J_{m}(\beta) I_{c}(n)
\end{aligned}
$$

[here $I_{s}(n)$ and $I_{c}(n)$ are given by Eq. (8)]. Note that the effect of rf field is to introduce the Bessel function $J_{m}(\beta)$ and to shift the frequency $\omega$ to $\tilde{\omega}_{m}=\omega+m \Omega$. For $\eta_{r f}$ $=0\left[J_{m}(0)=\delta_{0, m}\right]$ the expressions in Eqs. (24) and (25) reduce to the ones in Eqs. (6) and (7). The $I V$ characteristic and the locking ranges in current of the phase-locked steps follow from the dc part of Eq. (21)

$$
\eta-\alpha \omega=\overline{\left\langle\Psi \cos \left(\Phi_{0}\right)\right\rangle}+\overline{\left\langle\sin \left(\Phi_{0}\right)\right\rangle}
$$

It is important to note that the first average on the right-hand side of this equation is related to the flux-flow part of the $I V$ curve (it is different from zero mainly for $\tilde{\omega} \neq 0$ ) while the second average is related to phase locking (it is different from zero only for $\tilde{\omega}=0$, i.e., for $\omega=M \Omega$ ).

Assuming $\tilde{\omega} \neq 0$, we can compute

$$
\begin{aligned}
\overline{\left\langle\Psi \cos \left(\Phi_{0}\right)\right\rangle}= & \frac{1}{2 L} \sum_{n=0}^{\infty} \sum_{m=-\infty}^{\infty}\left[A_{n, m} \widetilde{I}_{c}(n, m)\right. \\
& \left.-B_{n, m} \widetilde{I}_{s}(n, m)\right] .
\end{aligned}
$$

The flux-flow part of the $I V$ characteristic follows from the balance between the dc part of Eq. (28) and $\alpha \omega-\eta$, this giving 


$$
\eta=\alpha \omega+\frac{1}{2} \sum_{n=-\infty}^{\infty} \sum_{m=-\infty}^{\infty} J_{m}^{2}(\beta)\left(\frac{\Gamma L}{\Gamma L+k_{n} L}\right)^{2} \frac{\sin ^{2}\left(\frac{\Gamma L-k_{n} L}{2}\right)}{\left(\frac{\Gamma L-k_{n} L}{2}\right)^{2}} \frac{\alpha \tilde{\omega}_{m}}{\left[\left(\tilde{\omega}_{m}^{2}-k_{n}^{2}\right)^{2}+\alpha^{2} \tilde{\omega}_{m}^{2}\right]} .
$$

In deriving this expression we substituted Eq. (28) into Eq. (27) and used Eqs. (24)-(26), together with the assumption $\tilde{\omega} \neq 0$. It is worth to note that Eq. (29) is independent of the phase angle $\theta_{1}$ and it has vertical asymptotes for $\omega=m \Omega$, $m=0,1,2, \ldots$. These singularities have no direct physical meaning [Eq. (29) was derived under the assumption $\omega$ $\neq M \Omega]$; however, they reflect the presence of phase locking (see below).

Assuming $\omega=M \Omega$, we have that

$$
\overline{\left\langle\sin \left(\Phi_{0}\right)\right\rangle}=J_{M}(\beta) \frac{\sin \left(\frac{\Gamma L}{2}\right)}{\frac{\Gamma L}{2}} \sin \left(\theta_{1}+\frac{\Gamma L}{2}\right)
$$

and the locking ranges follow, by changing the phase $\theta_{1}$ in the interval $[0,2 \pi]$, as

$$
\Delta \eta_{M}=2\left|J_{M}(\beta)\right| \frac{\sin \left(\frac{\Gamma L}{2}\right)}{\frac{\Gamma L}{2}} \mid
$$

It is interesting to note that the weight of the singularity at $\omega=M \Omega$ in Eq. (29) is proportional to the square of the above locking range and the vertical distance between the two hyperboliclike branches of the singularity is just $\Delta \eta_{M}$. We also remark that Eq. (31) predicts the elimination, at least to first order, of phase lock if $\Gamma L$ is a multiple of $2 \pi$. It

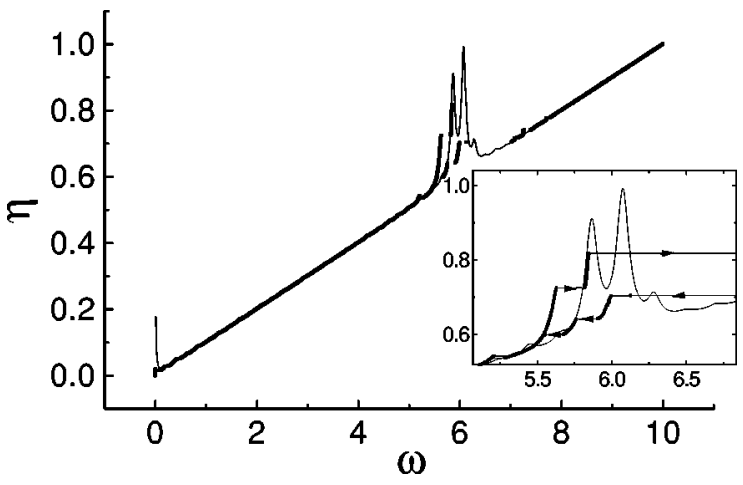

FIG. 1. IV characteristic of a long Josephson junction in the absence of microwave fields $\left(\eta_{r f}=0\right)$ for parameter values $\alpha$ $=0.1, L=15$, and $\Gamma=6$. The inset shows an enlargement of the flux-flow resonance at $\omega=\Gamma$ with the appearance of hysteretic phenomena. The thin curve refers to the analytical expression in Eq. (13) while the thick curve is obtained from numerical integration. should be noted, however, that a contribution to phase lock can also come from the resonant part of Eq. (28) (this is negligible if $\Psi$ is small, i.e., if the $I V$ curve is close to the Ohmic line). Results on the locking ranges valid for $M=1$ and in the limit $\Gamma / L \gg 1$ were also derived in Ref. 10 by a power balance analysis (note that here we have no restrictions on $M$ and $\Gamma / L)$. Finally, we note that in the considered case $\left(\eta_{r f} \neq 0\right),(\alpha / \omega)\left\langle\overline{\Psi_{t}^{2}}\right\rangle \neq\left\langle\overline{\Psi \cos \left(\Phi_{0}\right)}\right\rangle$, so that the power balance analysis of Sec. II is not useful to derive the fluxflow part of the $I V$ characteristic.

\section{NUMERICAL EXPERIMENT}

In order to check the analytical results derived above, we have performed a direct numerical integration of Eq. (1) subject to the boundary conditions in Eq. (2). For fixed values of magnetic field, damping constant, amplitude, and frequency of the rf field and length of the junction, we computed the normalized average voltage across the junction $V=\overline{\left\langle\Phi_{t}\right\rangle}$ as a function of the bias current $\eta$, taking as initial condition $n$ $=\Gamma L / 2 \pi$ fluxons equally spaced along the junction. The numerical $I V$ characteristic was obtained by integrating Eq. (1) long enough to eliminate all transients and measuring for each value of the bias current $\eta$ the corresponding voltage $V \equiv \omega$. To trace the curve, the bias current was increased in small steps from $\eta=0$ to 1 and then back to zero. At each $\eta$ step the final configuration of the field in the junction was used as initial condition for the next $\eta$ step.

In Fig. 1 we report the $I V$ characteristic of a long Josephson junction in the absence of microwave fields $\left(\eta_{r f}=0\right)$ for parameter values, $\alpha=0.1, L=15$, and $\Gamma=6$. We see the ap-

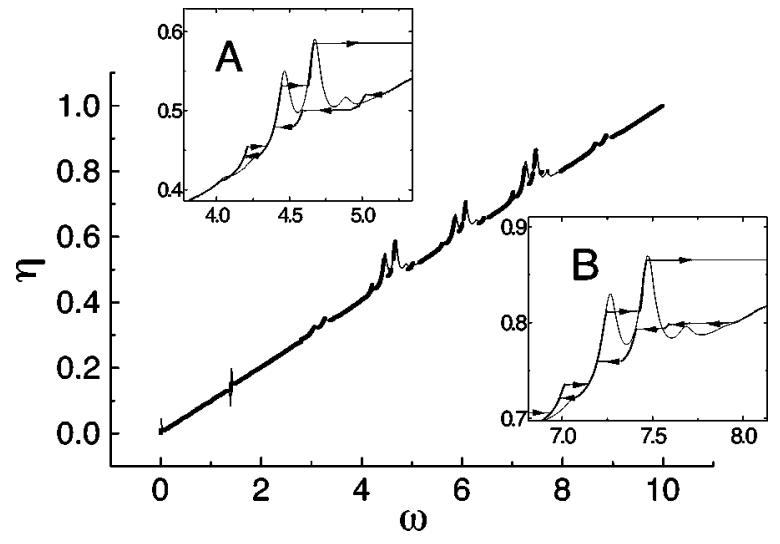

FIG. 2. IV characteristic of a long Josephson junction in the presence of a microwave field of amplitude $\eta_{r f}=3.0$ and frequency $\Omega=1.4$ for parameter values $\alpha=0.1, L=15$, and $\Gamma=6$. Insets A and B show an enlargement of the satellite steps at $\omega=\Gamma-\Omega$ and $\omega=\Gamma+\Omega$, respectively. The thin curve refers to the analytical expression in Eq. (29), while the thick curve is obtained from numerical integration. 


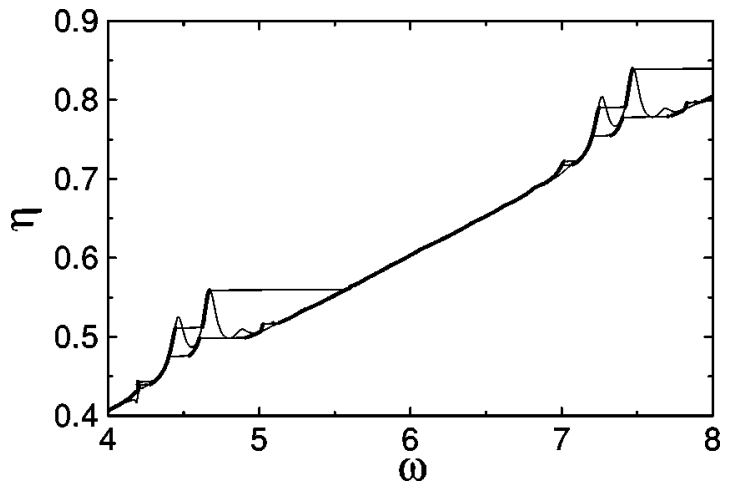

FIG. 3. Same as in Fig. 2, but for the rf-field amplitude $\eta_{r f}$ $=5.0$.

pearance of a flux-flow step at $\omega=\Gamma$ consisting of a few Fiske substeps spaced by $\pi / L$. Here the thin curve refers to the analytical expression in Eq. (13), while the thick curve represents the numerical results. The inset of the figure shows the flux-flow step in more detail, from which we also see the appearance of hysteretic phenomena (arrows show the direction of switching). Note that two of the numerical Fiske substeps appear broken due to our unidirectional procedure of swiping the bias current. From this figure it is evident that, except for stability problems (not included in our analysis), the two curves overlap in most of the plot, giving a good qualitative agreement between analytical and numerical results. This agreement becomes even better in the presence of an external microwave field as shown in Fig. 2, where the $I V$ curve of a junction with the same parameter values of Fig. 1 but with an applied rf-field of amplitude $\eta_{r f}=3.0$ and frequency $\Omega=1.4$, is reported. As before, the thin line represents the analytical $I V$ characteristic [Eq. (29)], while the thick one represents the numerical results. From this figure we see the appearance of satellite steps around the main flux-flow resonance of Fig. 1, in agreement with our analysis (small second satellite steps at $\omega=\Gamma \pm 2 \Omega$ are also visible). The insets of Fig. 2 show the first two satellite steps at $\omega=\Gamma \pm \Omega$ in more detail. By increasing the amplitude of the microwave field, Eq. (29) predicts a $J_{m}(\beta)^{2}$ modulation of the flux-flow resonance with a total suppression of the step for appropriate values of $\eta_{r f}$. This is actually what we find, as reported in Fig. 3, where the numerical and the analytical $I V$ curves are shown for the same parameter values used before, but for $\eta_{r f}=5.0$. We see that the two satellites are still present, but the main resonance at $\omega=\Gamma$ has almost disappeared. The number of satellite steps which can appear in the $I V$ characteristic depends on the parameters of the junction, and increases with the amplitude of the rf field. This suggests the possibility of using the flux-flow oscillator as a mixer device for high-frequency electronics (i.e., one can pump a signal at frequency $\Omega$ and detect it at a frequency $\Gamma \pm m \Omega$ ).

Before closing this section we check Eq. (31) for the phase-locked steps. This is done in Fig. 4, where the locking ranges vs $\eta_{r f}$ are reported for the first $M=0,1$, and 2 phaselocked steps. Here the thin line represents Eq. (31), while the circles, squares, and triangles denote numerical results for $M=0,1$, and 2 (the dotted lines were drawn just to help the

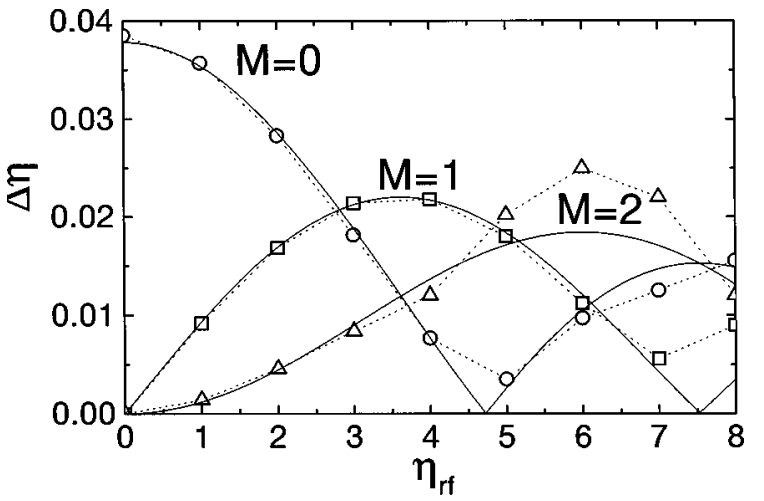

FIG. 4. Locking ranges in current vs the amplitude of the applied microwave field for the first three phase locked steps. The solid curves refer to the analytical expression in Eq. (31), while the circles, squares, and triangles denote numerical results for, $M=0$, 1 , and 2 , respectively (the dotted lines were drown to easily distinguish the different cases).

eye to distinguish the different cases), respectively. We see that there is a good agreement between analysis and experiment even for higher values of $\eta_{r f}$ and the deviation, especially for $M=2$, is due to mixing with the flux-flow structure. We also checked the $I V$ characteristic of a junction for values of the magnetic field for which Eq. (31) predicts a suppression of the phase-lock. We found that phase lock is strongly reduced (to first order) but not completely suppressed. These effects are ascribed to second-order (mixing) contributions which were neglected in our analysis.

\section{CONCLUSIONS}

We have developed a simple theory for a long Josephson flux-flow oscillator in the presence of spatially homogeneous microwave fields, which accounts for the appearance of phase locking and satellite steps around the flux-flow resonance. As a result we derived an analytical expression for the $I V$ characteristic of the oscillator in terms of an infinite series, the $m$ th term of which is modulated by the Bessel function of order $m$. This expression predicts the appearance of satellite steps around the flux-flow resonances at all the harmonics of the external rf frequency. We also derived the locking range in current for the phase-locking steps as a function of the junction parameters. We showed that by changing the amplitude of the rf field one can suppress the flux-flow resonance, and, for values of $\Gamma$ and $L$ such that $\Gamma L=2 p \pi, p \in N$, phase-locking phenomena are strongly reduced (at least to first order). These analytical results were found to be in excellent agreement with direct numerical integrations of the system, this being a confirmation of the validity of our approach. The extension of the above analysis to the case of rf fields applied through boundary conditions is presently under investigation.

\section{ACKNOWLEDGMENT}

One of us (M.S.) wishes to acknowledge financial support from the INFM (Istituto Nazionale di Fisica della Materia). 
*Also at Istituto Nazionale di Fisica della Materia (INFM), Unità di Salerno.

${ }^{1}$ T. Nagatsuma, K. Enpuku, F. Irie, and K. Yoshida, J. Appl. Phys. 54, 3302 (1983); 56, 3284 (1984).

${ }^{2}$ A. V. Ustinov, J. Mygind, and V. A. Oboznov, J. Appl. Phys. 72, 1203 (1992).

${ }^{3}$ A. V. Ustinov, J. Mygind, N.F. Pedersen, and V.A. Oboznov, Phys. Rev. B 46, 578 (1992).

${ }^{4}$ O.H. Olsen, A.V. Ustinov, and N.F. Pedersen, Phys. Rev. B 48, 13133 (1993).

${ }^{5}$ V.P. Koshelets, A.V. Shchukin, S.V. Shitov, and L.V. Filippenko, IEEE Trans. Appl. Supercond. 3, 2524 (1993).

${ }^{6}$ Y.M. Zhang and D. Winkler, IEEE Trans. Microwave Theory Tech. 4, 726 (1994).

${ }^{7}$ M. Cirillo, F. Santucci, P. Carelli, M.G. Castellano, and R. Leoni,
J. Appl. Phys. 73, 8637 (1993).

${ }^{8}$ A. Barone and G. Paternò, Physics and Applications of the Josephson Effect (Wiley, New York, 1982).

${ }^{9}$ M. Cirillo, N. Grønbech-Jensen, M.R. Samuelsen, M. Salerno, and G. Verona Rinati, Phys. Rev. B 58, 12377 (1998).

${ }^{10}$ N. Gronbech-Jensen and M. Cirillo, Phys. Rev. B 50, 12851 (1994); N. Grønbech-Jensen, P.S. Lomdahl, and M. Cirillo, Phys. Rev. B 51, 11690 (1995).

${ }^{11}$ R. D. Parmentier, in The New Superconducting Electronics, Vol. 251 of NATO Advanced Study Institute, Series E: Applied Sciences, edited by H. Weinstock and R. W. Ralston (Kluwer, Dordrecht, 1993), pp. 221-248.

${ }^{12}$ D.W. McLaughlin and A.C. Scott, Phys. Rev. A 18, 1652 (1978).

${ }^{13}$ R.E. Eck, D.J. Scalapino, and B.N. Taylor, Phys. Rev. Lett. 13, 15 (1964). 